\title{
EXPOSÉ ET DISGUSSION DES TRAVAUX LES PLUS RÉCENTS RELATIFS AUX ÉCHELLES A POISSONS
}

Par M. SABATIER DE LACHAI)ENËDE

Conservateur des Eaux et liorits.

(Suile)

Arrivons maintenant an travail inédit de M. Monis, qui n a pas les mêmes conceptions que M. Scumssuax. Il n'étudie, d'ailleurs, que le Saumon, alors que le prolesseur suisse examine le problème plus général du l'oisson.

V. Moné estime que le Saumon surmonte les vbstacles soit en nagreant, soit en sautant, pourvu qu'il ne soit pas astreint a fournir un effort-limite, dont il sagit d'exprimer la valeur. Et, pour cette recherche, on ne peut utiliser que ce qu'on voit : le saut.

La détermination effectuée, il sera loisible de sommeltre à une discussion rationnelle les dispositifs pour l'ulilisation de cet effort, destinés an franchissement des barmages et, enfin, les meilleurs moyens d'attirer le saumon au pied de ces dispositifs.

Ie saut a fait, de la part de M. Mons:r, lohjel d'observalions très atten-

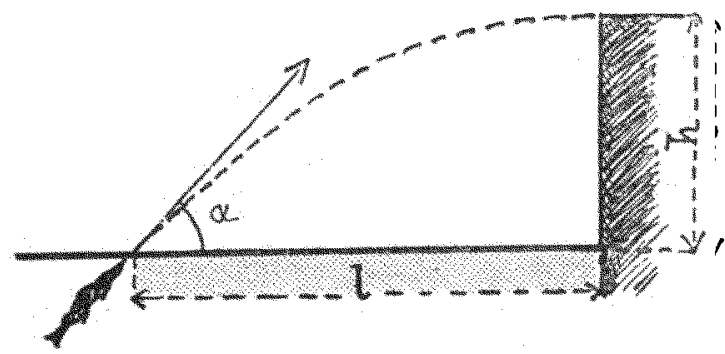

Fig. 12. - Siut du Situmon. tives. Le Saumon incline d'abord son corps dans la direction de la crête de l'obstacle qu'il veut franchir, puis prend son élan.

A linstar d'un projectile, son corps, animé d'une vitesse initiale $V_{0}$ décrit hors de l'eau une parabole(voir fiğr. I $)$; on connait, en conséquence, Ja relation entre cette vitesse, l'angle d'inclinaison de la trajectoire à son origine et la hauteur $h$ du sommet de la courbe au-dessus de l'horizontale (h est, en fait, la hauteur de l'obstacle) ; on a en effet:

$$
\mathrm{h}=\frac{\mathrm{V}_{\mathrm{o}^{2}} \sin ^{2} \alpha}{2 \mathrm{~g}}
$$

Article available at http://www.kmae-journal.org or http://dx.doi.org/10.1051/kmae:1929003 
La demi-longueur I de la corde qui sous-tend la parabole, c'est-à-dire la distance du point d'émergence du Poisson au pied de l'obstacle, est donnée par la formule :

Substituant :

$$
\mathrm{I}=\frac{\mathrm{V}_{0}{ }^{2} \operatorname{Sin} \cdot \alpha \operatorname{Cos} \cdot \alpha}{\mathrm{g}}
$$

$$
\mathrm{l}=-\frac{2 \mathrm{~h}}{\operatorname{Tg} \cdot \mathrm{x}}
$$

D'oì l'on déduit que, pour un barrage déterminé, I sera d'autant plus court que a sera plus grand.

Pratiquement, le Saumon, en sautant, s'arrange pour que la distance soit voisine de 2 fois la hauteur de l'obstacle.

Alors $\alpha$ est voisin de $4^{\circ}$, on peut poser $\operatorname{Sin} .{ }^{2} \alpha=1 / 2$, d'où :

$$
\mathrm{V}_{\bullet}=\sqrt{\mathbf{4} \mathrm{g} \mathrm{h}}
$$

En admettant le saut moyen à $I^{\mathrm{m}} 25$ de hauteur, $V_{0}=7$ mètres-secondes environ.

Telle serait la vitesse initiale du Saumon type et les courants qu'il peut remonter seraient ceux inférieurs à $7 \mathrm{~m}$.-s. ; au-delà il serait entraîné ou bien resterait sur place. D'autre part, la vitesse maxima de l'eau dans une passe devra être, par excmple, de $3^{\text {m }}$ 'o ì la seconde pour que le Saumon la puisse franchir avec une vitesse relative de $3^{\mathrm{m}} 50$.

La vitesse limite du courant étant ainsi déterminée, M. Moncr eraminant la question de la hauteur maxima à donner aux échelles à Poissons, estime que celle-ci n'est limitée par rien autre que par des motifs d'ordre financier. Il suffirait d'aménager, sur le parcours de ces échelles, en suffisance, des bassins de repos, pour que le Poisson puisse franchir n'importe quelle hauteur. Alors, il s'élance et monte rapidement, s'arrêtant aux paliers un temps plus ou moins long pour continuer ensuite son ascension : jamais il ne reviendrait en arrière.

Des observations faites à Kjukanföss (Norvège) montrent que 26 mètres de hauteur, 285 mètres de longueur et zoo coudes n'effrayent pas le Saumon de ces régions.

Dans les passes, l'eau est blanche si elle est écumeuse, verte si elle n'est pas émulsionnée d'air. Le Saumon préfère les eaux vertes, où il voit clair et où il peut se diriger.

Pour qu'une passe puisse c̀tre franchie, il faut que l'entrée en soit d'un accès facile.

Les quelques indications suivantes peuvent guider pour leur installation.

D'abord, le Saumon recherche les masses d'eau en mouvement. Plus l'accès d'une échelle se confondra avec le courant principal de la rivière, plus elle sera facile ì trouver.

Dans un courant rapide, un abri de repos est nécessaire ; l'entrée d'une 
passe sera donc bien placée au milieu d' un goullre oì le Saumon viendra stationner.

Le bruil parait exercer une altirance ; tel celui de l'eau tombant en cascades.

Un éclairage intense, lia nuit, semble aroir une influence favorable, sans que le fait soit nettement démontré.

Enfin, les entrées à coloration foncée seraient plus facilement repérées que celles de teinte claire, et la rusticité de leur aménagement est recommandable, comme imitant la nature.

La théorie hydraulique des diflérents dispositif's de tranchissement des obstacles fait ensuite l'objet d'une étude spéciale de M. Moner dont voici le résumé :

$I^{\circ}$ Les simples coursiers à parois rugueuses unt une vitesse de l'eau généralement trop forte, savoir :

$$
\begin{aligned}
& \text { Pour une pente de } 10 \% \text {, de } 5 \text { m. jo par seconde } \\
& \text { - } \quad-\quad \begin{array}{l}
20 \%,-7 \mathrm{~m} .60 \quad- \\
-\quad 30 \%,-9 \mathrm{~m} .40 \quad-
\end{array}
\end{aligned}
$$

'Tous ces chiffres étant supéricurs à la vitesse adoplée de $3 \mathrm{~m}$. jo, il résulte que ces dispositifs ne devront être employés que sur des sections à longueur assez petite pour limiler l'effort à faire au moindre temps possible.

$2^{c}$ Les coursiers à chicane sont recoupés par des cloisons grâce auxquelles est allongé le parcours, donc atténuée la pente ; la multiplication des surfaces de froltement a aussi pour eflet d'amortir la vitesse du courant par des chocs contre les parois.

$3^{\circ}$ L'échelle "Caméré " est formée d'un couloir incliné dont les côtés et le fond sont percés de feriles par lesifuelles l'eau d'amont est injectée sous pression. Il en est plusicurs types, peu intéressants en raison de la grande consommation d'eau; les résultats obtenus ont, d'ailleurs, été médiocres.

$4^{\circ}$ Les coursiers à amortisseurs du mudèle "Denil " sont plus avantageux que les précédents, car ćest l'eau du coursier lui-même qui est utiliséc pour freiner l'accélération due à la pesanteur.

L'amortisseur le plus simple est constitué de deux plans perpendiculaires au fond du coursier, formant entre enx un angle dièdre de $90^{\circ}$ et inclinés à $45^{\circ}$ sur les parois latérales. Ces plans sont échancrés de manière à laisser passage au courant.

La réduction de la vitesse du courant qui dévale est due aux frottements, aux remous, à l'aboorption partielle de l'énergie cinétique par les masses d'eau tourbillonnantes.

$5^{\circ}$ Les cuves étagrées à orifices noyés comportent une série de bassins en gradins en communication par des ouvertures situées au-dessous du niveau liquide. L'eau débitée par elles rencontre, dans la cuve suivante, 
des veines liquides animées d'une vitesse moindre. Il se produit alors des frottements ; de plus, le courant s'épanouissant, à la sortie de l'orifice, sa vitesse diminue el climinuera d'autant mieux que la distance séparant deux cloisons successires sera plus grande. Ce syslème est à adopter, sur les rivières sujettes à varialions de niveau, pour la partie amont des passes, mais il ne convient pas pour leur partie aval, parce que les orifices, se trouvant immergés à trop grande profondeur au moment des crues, l'eau qui s'en écoule ne provoque plas lappel du Poisson.

$6^{\circ}$ Les vannes de fond te sont autre chose que des orifices noyés. A la poudreric nationale de Pont-de-Buis il en est une, traversée maintes fois par le Saumon, nonobstant une différence de niveau de j mètres. La vilesse du courant, donnéc par la formule $v=\sqrt{2 \mathrm{gh}}$ est de 9 m. $9^{\circ}$; celle des Poissons franchissant cetle vanne doit itre ainsi de 11 mètres environ.

$7^{\circ}$ Les cuves étagées à dévcrsoirs, qui ont des applications nombreuses en Norvège, ne fonctionneat bien que quand la différence de niveau d'une cuve à l'autre ne dépasse par 0,25 à 0,30 . La passe Cockemouth (Cumberland), qui donne de bons résultats, a été construite sur ce principe.

$8^{\circ}$ Les cuves étagées à cascades ne peuvent ètre franchies par le Poisson qu'en sautant. Beaucoup de sujets se dépensent en efforts inutiles; après Ii à 20 essais, fatigués, ils se laissent aller au fil de l'eau.

$9^{\circ}$ Les passes rustiques sont formées le bassins communiquant entre eux par des déversoirs noỵés, par des coursiers à parois rugueuses ou par de pelites cascales íreusées dans le sol. ces passes comprennent le minimum dourage dart.

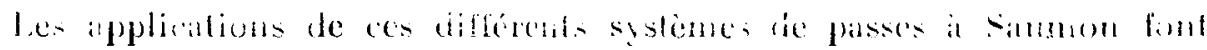

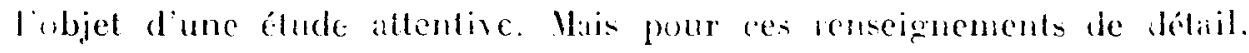
anus renverrous le lecteur à lourage mime.

Faisant maintenant applicalion des données de MY. Scmmassunin et Monel et passant de la théorie à la pratique, nous constatons qu'un courant d"une vilesse de $i, i$, à la seconde pourra ôtre vaincu par les Saumons, mais ne le sera pas par les autres Poissons qui ne peuvent remonter plus de $2 \mathrm{~m}$.-s. cnviron.

Comme une échelle doit être utilisable par tous les Poissons, nous sommes conduits à établir les projets en adoptant cette dernière vitesse comme un maximum. Mais ceci a, évidemment, l'inconvénient d'impliquer pour la passe un développement considérable avec un prix correspondant.

Les échelles qui semblent, généralement, convenir le mieux, à s'en rapporter à l'expérience de M. Schmassmaxx, sont celles constituées de bassins étagés en communication par orifices noyés.

Ces échelles sont, théoriquement, très satisfaisantes. Toutefois, comme 
le fait observer M. Monel, sur les rivières sujettes à amples variations de niveau, elles sont d'efficacité réduite au moment des crues.

Il conviendra, en pareille conjoncture, de recourir à l'échelle mixte formée pour la partie amont d'une passe à orifice nové, pour la partie aval d'un coursier amortisseur ; elle se prètera bien à toutes les variations de niveau.

Il semble même, en définitive, que ce dispositif doive ètre considéré comme étant, d'une façon geénérale, celui auquel devront aller les préférences. Bref, sauf cas exceptionnels, l'échelle la plus avantageuse serait composée, en amont de cuves étagées à orifices noyés, en aval d'un coursier "Denil".

La vitesse de l'eau étant arrêtée à 2 mètres à la seconde, la différence de niveau de l'eau entre les cuves sera donnéc par la formule :

$$
\mathrm{h}=\frac{\mathrm{V}^{2}}{2 \mathrm{~g}}=\frac{4}{19,62}=0^{\mathrm{m}} 20 .
$$

Les ouvertures dans les parois seraient en ligne droite pour ne pas exposer le Poisson à aller heurter la cloison d'amont d'un compartiment en poussant droit devant lui après avoir traversé l'orifice de la paroi d'aval.

Reste le choix de l'emplacement de l'échelle.

Sur ce point, il y a désaccorl entre les deux spécialistes dont nous avons analysé les récents travaux.

D'après MI. Morth, le Saumon ne s'engagera dans une passe que s'il a l'impression qu'il n'abandonne pas le cours de la rivière. En outre, plus l'entrée se confondra avec le courant principal, micux cela viudra.

Estimant, au contraire, (que le Poisson est un fervent partisan de la théorie du moindre efiort, $\|$. Simussmax pense quil remontera le courant en suivant les bords où la vitesse de l'eau est moindre. Ciest donc là qu’il faudrait placer, de préférence, de pied de l'échelle.

Cette divergence est d'importance, alors surtout que la cause essentielle du non-fonctionnement de bien des échclles parait bien ètre leur mauvaise implantation. On est amené à se demander si les Poissons en instance de remonte se comportent tous de la mème manière au seuil d'un olstacle dressé sur leur chemin.

Lne étude expérimentale dé cette question serait assurément des plus intéressantes. En attendant, il serait peut-être prudent de ne pas ficer les pieds des échelles d'une maniere invariable ; il ne semble pas impossible ¿ie prévoir, avant la construction définitive, l’installation d'une passc yrovisoire facilement déplaçable.

Il nous reste à comparer les méthodes SCuMissuanx et Morri. en ce qui concerne la détermination le l'effort-limite des divers Poissons et, par suite, celle de la vitesse maxima du courant qu'ils peuvent remonter.

Certaines obscrvations frappent le lecteur de ces deux méthodes. Pour M. Scimassuax, la mage est le seul morle de propulsion. W. Mokria admet 
le saut au même titre que la nage. Dans ce cas le Poisson quitte le milieu liquide pour passer dans un autre dont la résistance est toute différente. On s'explique alors mal que, de la hautcur du saut dans l'air, on puisse déduire la vitesse de nage dans l'eau. Sur ce point il semble bien que la méthode donne prise à quelques critiques, théoriquement au moins.

M. Schmssmave utilise passivement les Poissons qu'il veut étudier ; il les pèse, puis les tire au bout d'un fil, se préoccupant uniquement de détermincr là résistance opposée par l'eau au mouvement du sujet en expérience.

M. Morel observe des Saumons actifs et sautant. Il évalue la hauteur du bond, sans s'inquiéter du poids de l'animal ni de l'énergie interne qu'il développe, il en déduit l'élan nécessaire pour l'élévation à une hauteur donnce, et par suite, la vitesse dont il cst accidentellement capable.

Le saul moyen du Saumon moven est alors, pour M. Monel, une sorte de synthèse des différents élérnents dont M. Scumassmann examine un en purliculier, de façon attentive, en raison de son importance prépondérante.

Nous conclurons de ces remarques que la méthode Schmassmann nous paraît rigoureusement scientifique, avec un résultat exact pour chaque poisson étudié, alors que la méthode Morec moins précise, ne donne, pour le seul Saumon, qu'une approximation, mais suffisante, semble-t-il, pour les besoins de la pratique.

ll serait, assurément, du plus grand intérèt de voir si les constatations faites, de part et d'autre sont ou non concordantes.

Mais, M. Schmassmanv n`a étudié qu'un seul Saumon, du poids de 4 k. 660 ; encore considère-t-il comme suspectes les valeurs par lui enregistrées des résistances à l'entraînement à diverses vitesses. Il est très regrettable que les expériences n'aient pas été plus nombreuses et plus poussées. Il serait indiqué de les reprendre et compléter.

Quoi qu'il en soit, MM. Morrec et Scimmassmanin ont alordé, par des voies différentes, le problème fondamental des échelles à poissons.

Nul doute qu'en continuant les recherches par eux inaugurées, on n'arrive à des données précises sur l'aptitude des Poissons à vaincre la résistance d'un courant ; alors les échelles pourront être rationnellement construites, ce qui est la condition formelle de leur efficacité.

Qu'il nous soit permis, en terminant, d'adresser tous nos remerciements a MM. Schmassmany et Morec. qui ont bien voulu nous fournir les éléments de celte communication ; ainsi nous ont-il mis à même d'attirer sur leurs remarquables et récents travaux l'attention de ceux que préoccupent les conséquences, pour le peuplement des cours d'eau, de l'exploitation toujours plus intensifiée de la houille blanche ou verte. 